\title{
DEMOGRAFÍA Y ECONOMÍA DE UNA CAPITAL ESTATAL. MEXICALI, BAJA CALIFORNIA
}

\author{
Por \\ Gabriel Estrella Valenzuela* \\ Arturo Ranfla González**
}

\begin{abstract}
RESUMEN
Este trabajo tiene como objetivo central, identificar de manera general los sectores de actividad económica en los que la ciudad de Mexicali presenta un grado de especialización respecto del conjunto del estado de Baja Califomia. Para lograr ese objetivo, en la primera sección se señalan las características generales que han definido las tendencias del comportamiento demográfico de esta zona del país a lo largo del siglo XX, con el propósito de hacer evidente el nexo que ha estado presente entre las modalidades que ha asumido el desarrollo económico de Mexicali y las fluctuaciones que se han observado en los componentes de su dinámica poblacional. En la segunda sección del trabajo, se aborda la evaluación de la especialización de la estructura económica de Mexicali, en términos de la capacidad de generación de empleos y del tipo de empleos generados. En este caso, se recurre a la perspectiva analítica de la "base económica", dado que interesa destacar el papel que ha desempeñado la contigüidad a la frontera con los Estados Unidos, respecto de las posibilidades - pretéritas y actuales - de desarrollo de la economía de esta localidad a través de su capacidad de respuesta a la demanda externa de bienes y servicios.
\end{abstract}

\begin{abstract}
This work has as a main objective, to identify, in a general way, the sectors of the economical activity in which the city of Mexicali presents a grade of specialization in respect to the state of Baja California in general. To accomplish such objective, in the first part, the general characteristics that have defined the tendencies of demographic behavior in this zone of the country through the $20^{\text {th }}$ century are marked, with the purpose of making evident the nexus that has existed between the modalities that have assumed the economical development of Mexicali, and the fluctuations that have been observed in the components of its population dynamic. The second part of this work is about the evaluation of the specialization of the economical structure of Mexicali, related to the capacity of creating jobs, and the kind of jobs that are created. In this case, tha analitic perspective of the "economical basis" is used, since it's important to show the role the border with the United States has played, in relation to the possibilities - past and present - of development in the economy of this area through the capacity of responding to the external demand of goods and services.
\end{abstract}

* Investigador del Instituto de Investigaciones Sociales, UABC. Investigador Nacional y Becario UABC al Desempeño. E-mail: gestrell@faro.ens.uabc.mx

** Investigador del Instituto de Investigaciones Sociales, UABC. E-mail: aranfla@faro.ens.uabc.mx

N.del E. Este artículo se aprobó en mayo de 1997 para su publicación en Estudios Fronterizos. 


\section{INTRODUCCIÓN}

A lo largo de este siglo, las seis entidades fronterizas del norte de México (Baja California, Chihuahua, Coahuila, Nuevo León, Sonora y Tamaulipas) han compartido al menos tres elementos comunes en relación con su desarrollo socioeconómico. El primero de esos elementos ha consistido en que esas seis entidades se han caracterizado por presentar un crecimiento poblacional superior al del total del país, pues de 1930 a 1990 los 38 municipios fronterizos que colindan con los Estados Unidos superaban los ritmos de crecimiento de las entidades a las que pertenecen (Zenteno y Cruz, 1988:399-423). Esos elevados ritmos de crecimiento que han caracterizado a esta zona del país y a sus municipios fronterizos, han dado lugar al segundo elemento común que comparte esta zona del país, el cual consiste en los elevados niveles de urbanización que ha asumido la población que en ellos reside (Gutiérrez, 1994).

Los dos rasgos anteriores - la acelerada dinámica demográfica y la urbanización de la población fronteriza del norte de México- han estado sustentados en el tercer elemento que han compartido estas zonas del país, dado que ha consistido en su capacidad para presentar ritmos de desarrollo económico relativamente superiores a los de la mayoría de otras entidades, los que se han traducido en niveles de bienestar comparativamente mejores para la población que ahí reside (CONAPO, 1992).

En el trabajo que se presenta a continuación, se analiza la trayectoria demográfica y las tendencias de la estructura productiva de la ciudad de Mexicali, Baja California, con el proposito de señalar tanto el nexo que ha vinculado las modalidades que ha asumido el desarrollo económico de la ciudad con las variaciones de su dinámica poblacional, como la especialización de su estructura económica, ya que que interesa destacar el papel que desempeña la frontera con los Estados Unidos, respecto de las posibilidades - pretéritas y actuales- de desarrollo de la economía de esta localidad a través de su capacidad de respuesta a la demanda externa de bienes y servicios.

\section{CARACTERÍSTICAS DEMOGRÁFICAS}

Desde la perspectiva estrictamente demográfica, puede afirmarse que tanto Baja California como su capital estatal, Mexicali, comparten el rasgo de ser áreas del territorio nacional que se encontraban virtualmente deshabitadas al inicio del siglo XX. Al respecto, los datos presentados en el cuadro 1 permiten advertir que el primero de los 10 registros censales de este siglo, consignó a menos de 8000 residentes en Baja California, y que para 1900, 
tanto el municipio como la ciudad de Mexicali, aún no aparecen como entidades censables.

Otro elemento común que han compartido Baja California y Mexicali en materia de población a lo largo de este siglo, es el relativo al acelerado ritmo con el que han visto incrementar el total de residentes que habitan en esta zona del país. En ese sentido, la información contenida en el cuadro mencionado indica que durante las tres primeras décadas, mientras el estado incrementaba su población más de 6 veces (de 7583 a 48 327), los residentes de Mexicali se multiplicaban por 32 (de 462 a 14 842); de 1930 a 1960 la población de ambas áreas se incrementó más de 10 veces (a 520165 para el estado y a 174540 para la ciudad). En los tres últimos decenios, Baja California triplicó su población (con 1660855 en 1990), en tanto que la de Mexicali se multiplico por un factor de 2.5 al acumular más de 438000 habitantes en 1990.

\section{CUADRO 1. Estado de Baja California, municipio y ciudad de Mexicali (1900-1992).}

\begin{tabular}{|c|c|c|c|c|c|}
\hline \multirow[b]{2}{*}{$\begin{array}{l}\text { Fecha } \\
\text { censal }\end{array}$} & \multicolumn{5}{|c|}{ Población total por entidad y \% de participación } \\
\hline & $\begin{array}{c}\text { Baja } \\
\text { California }\end{array}$ & $\begin{array}{l}\text { Mpo.de } \\
\text { Mexicali }\end{array}$ & $\begin{array}{c}\% \text { del total } \\
\text { estatal }\end{array}$ & $\begin{array}{l}\text { Ciudad de } \\
\text { Mexicali }\end{array}$ & $\begin{array}{c}\text { Cd. \% del } \\
\text { mpo. }\end{array}$ \\
\hline 1900 & 7583 & 0 & & 0 & \\
\hline 1910 & 9760 & 1614 & 16.5 & 462 & 28.6 \\
\hline 1920 & 23537 & 14599 & 62.0 & 6782 & 46.5 \\
\hline 1930 & 48327 & 29985 & 62.0 & 14842 & 49.5 \\
\hline 1940 & 78907 & 44399 & 56.3 & 18775 & 42.3 \\
\hline 1950 & 226965 & 124362 & 54.8 & 64658 & 52.0 \\
\hline 1960 & 5201665 & 281333 & 54.1 & 174540 & 62.0 \\
\hline 1970 & 870421 & 396324 & 45.5 & 263498 & 66.5 \\
\hline 1980 & 1177886 & 510664 & 43.4 & 341559 & 66.9 \\
\hline 1990 & 1660855 & 601938 & 36.2 & 438377 & 72.8 \\
\hline $1992 *$ & 1908434 & 670624 & 35.1 & N.D. & \\
\hline
\end{tabular}

Fuente: Censos generales de población.

* Encuesta nacional de la dinámica demográfica, INEGI, 1992. Distribución municipal $1992=(\mathrm{i}=70 / 90 \%$ de B.C. $)$. 
Los dos rasgos antes mencionados, han sido acompañados por la presencia de dos tendencias de sentido divergente. La primera consiste en que, como lo muestran los datos en el cuadro 1 y su representación en la figura 1, desde 1930, el municipio de Mexicali ha venido reduciendo su participación en el total de la población estatal, debido a que desde esa fecha la velocidad de crecimiento de la población del primero ha sido inferior a la de la entidad en su conjunto.

La segunda tendencia, de sentido inverso a la anterior, consiste en la creciente participación que se observa de la población de la ciudad de Mexicali en el total municipal correspondiente. En este caso, como lo muestran las barras de la figura 1, la ciudad de Mexicali logró recuperar su trayectoria de crecimiento poblacional a tasas superiores a las municipales desde la década de los años cuarenta, y ha sostenido ese patrón durante la segunda mitad del siglo.

En conjunto, los rasgos y tendencias hasta ahora mencionados, permiten advertir que tanto en Mexicali como en Baja California, se han configurado tres periodos que caracterizan a sus respectivos comportamientos demográficos. Durante el primer periodo, que abarca de 1900 a 1940, se dio inicio al poblamiento de ambas áreas a partir de los flujos de inmigrantes que arribaron para colonizar las zonas que surgieron al ampliarse la frontera agrícola, mediante la derivación de las aguas del río Colorado hacia el valle de Mexicali. En el transcurso de la última de estas cuatro décadas, la ciudad de Mexicali presentó su tasa más baja de crecimiento total de población en lo que va del siglo, debido fundamentalmente al proceso de localización rural de residentes que implicó la implementación de la reforma agraria en esta zona del país a partir de 1937.

Ese proceso de reparto agrario iniciado a finales de los años treinta, se acompañó de un conjunto de acciones gubernamentales en materia de dotación de infraestructura (como lo fue la creación del distrito de riego y la construcción de la vía ferrocarrilera que permitió el inicio de la comunicación terrestre por territorio nacional con el interior del país), de política fiscal (al sustituir los perímetros libres con el régimen de zonas libres a la importación) y así como la económica (desde entonces se incluyó a Baja California en la zona económica con mayor salario mínimo diario), con las que se promovió el poblamiento de esta área de la frontera con los Estados Unidos.

La manifestación demografica del efecto que generaron ese conjunto de medidas de política pública, consistió en la reactivación de la velocidad de crecimiento de la población de la ciudad, del municipio y del estado durante un lapso que se prolongo a lo largo de las tres décadas comprendidas de 1940 a 1970 (figura 1). Durante este segundo periodo de la 


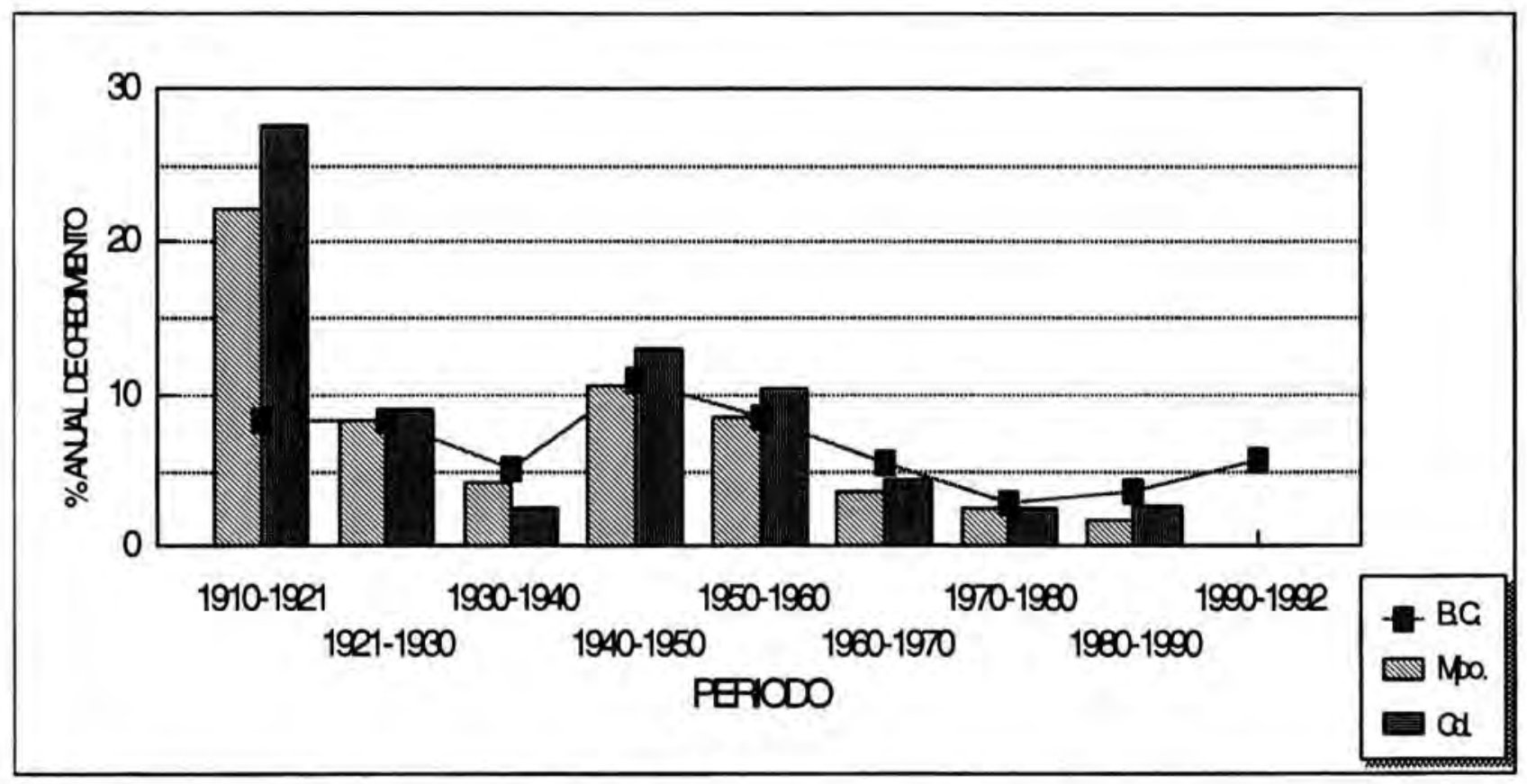

Figura 1. Baja California, municipio y ciudad de Mexicali. Tasa Anual de Crecimiento Total de la población, 1910-1990.

Fuente: Elaborada con datos del cuadro 1. 
trayectoria demográfica de esta zona, Mexicali se constituyó en un punto de atracción -al igual que otras entidades y localidades de la frontera norte de México- para la población que migraba desde el interior del país, buscando aprovechar las oportunidades generadas tanto en el mercado laboral internacional mediante la contratación en el "Programa Bracero", como en la demanda local de trabajo, que originaron la bonanza agrícola sustentada en el cultivo del algodón y sus efectos económicos multiplicadores, los que se ampliaron con el asiento de los poderes estatal y municipal en esta localidad, al constituirse Baja California en estado federado en 1953.

A partir de mediados de la década de los sesenta, Mexicali transitó por una coyuntura que modificó radicalmente su capacidad de atracción demográfica, dado que el finiquito del convenio internacional del "Programa Bracero" y el surgimiento de la crisis en la producción algodonera (por problemas fitosanitarios, de salinidad y precios internacionales), contrajeron las oportunidades de ocupación que tradicionalmente se habían sustentado en esas actividades.

Esa coyuntura que dio paso al inicio del tercer periodo de la trayectoria demografica de Mexicali (de 1970 a la fecha), se tradujo en un comportamiento poblacional que en principio parece mostrar evidencias de estar integrado por dos elementos característicos. El primero de esos elementos, como se puede observar en los datos presentados en el cuadro 2, consiste en una tendencia decreciente de los valores de los componentes del crecimiento natural de la población, tanto en el caso de Baja California como en el del municipio de Mexicali.

En efecto, los datos mencionados dan cuenta de un proceso de reducción paralela de la mortalidad y la natalidad en el orden de $30 \%$ durante el transcurso de las dos últimas décadas, el cual ha redundado en la homogeneización de los niveles de mortalidad y natalidad de Baja California y Mexícali, y en consecuencia en la igualación de la velocidad de su crecimiento natural.

Referente a esos dos rasgos característicos del crecimiento natural de la población en Baja California, conviene señalar que ambos han sido identificados como parte del perfil del comportamiento demográfico conjunto de las seis entidades fronterizas del norte del país. En ese sentido, dos estudios que recurren a procedimientos diferentes, han logrado establecer que en esas seis entidades, la reducción, tanto de la mortalidad como de la natalidad, se inició con antelación respecto al resto de México (Corona, 1991), y que por lo tanto, el proceso de la transición demográfica - de patrones de alta a baja mortalidad y fecundidad - ha avanzado más en esta zona del país (Monterrubio, Flores y Armada, 1993:299-314). 


\section{CUADRO 2. Estado de Baja California, municipio y ciudad de Mexicali (1960-1990).}

\begin{tabular}{|c|c|c|c|c|c|}
\hline \multirow[b]{2}{*}{$\begin{array}{l}\text { Entidad y } \\
\text { fecha }\end{array}$} & \multicolumn{5}{|c|}{ Indicadores de dinámica demográfica } \\
\hline & $\begin{array}{c}\text { T. Bruta } \\
\text { Natalidad }\end{array}$ & $\begin{array}{l}\text { T. Bruta de } \\
\text { Mortalidad }\end{array}$ & $\begin{array}{c}\text { T.de Crec. } \\
\text { Natural }\end{array}$ & $\begin{array}{c}\text { T. de Cre. } \\
\text { Total }\end{array}$ & $\begin{array}{l}\text { T. de Crec. } \\
\text { Social* }\end{array}$ \\
\hline \multicolumn{6}{|l|}{ Baja California } \\
\hline $1960-1970$ & 46.67 & 7.75 & 3.89 & 5.52 & 1.63 \\
\hline $1970-1980$ & 40.03 & 6.55 & 3.35 & 2.95 & -0.40 \\
\hline $1980-1990$ & 30.70 & 5.12 & 2.56 & 3.57 & 1.01 \\
\hline $1985-1990^{*}$ & & & & & 1.80 \\
\hline \multicolumn{6}{|c|}{ Municipio de Mexicali } \\
\hline $1960-1970$ & 45.98 & 7.49 & 3.85 & 3.64 & -0.21 \\
\hline $1970-1980$ & 38.75 & 6.12 & 3.26 & 2.46 & -0.80 \\
\hline $1980-1990$ & 30.92 & 5.26 & 2.57 & 1.69 & -0.88 \\
\hline $1985-1990^{*}$ & & & & & 2.15 \\
\hline \multicolumn{6}{|c|}{ Ciudad de Mexicali** } \\
\hline $1960-1970$ & 45.98 & 7.49 & 3.85 & 4.39 & 0.54 \\
\hline $1970-1980$ & 38.75 & 6.12 & 3.26 & 2.52 & -0.74 \\
\hline $1980-1990$ & 30.92 & 5.26 & 2.57 & 2.58 & 0.01 \\
\hline $1985-1990^{*}$ & & & & & 2.35 \\
\hline
\end{tabular}

Fuente: Censos generales de población. CONAPO: Registro de nacimientos y defunciones. Registro civil nacional, (1982-1991).

(*) TCs calculada como residual (TCT-TCN), y 1985-1990 con saldo inmigratorio censal.

(**) El crecimiento natural y sus componentes son los del municipio.

Por otra parte, el segundo elemento que parece caracterizar a este periodo de la trayectoria demografica de la ciudad y del estado, es el relativo a la defínición de un nuevo ciclo de reactivación de la velocidad del crecimiento social de su población. En relación con éste, los datos de crecimiento social del cuadro 2 permiten advertir que, mientras a nivel municipal se ha acentuando el rasgo de constituir un área de emigración neta a lo largo de la dos últimas décadas (al aumentar su tasa de crecimiento social anual de $-0.21 \%$ de 1960 a 1970 a $-0.88 \%$ entre $1980-1990$, tanto en el estado como en la ciudad de Mexicali se presentan evidencias de una recuperación de la capacidad de atracción de inmigrantes a partir de la década de los ochenta, en particular durante su segundo quinquenio.

Ese último rasgo del comportamineto demográfico de Mexicali y Baja California, en principio, puede asociarse a dos conjuntos de procesos de orden económico. El primero, es el relativo a los procesos de alcance regional que se originaron en la frontera norte del país, con la puesta en 
operación del Programa de Industrialización Fronteriza en 1965, ya que éstos modificaron las características de la estructura de oportunidades económicas al fomentar el desarrollo de la industria maquiladora de exportación, particularmente a partir de las sucesivas devaluaciones del peso mexicano que se iniciaron en 1976. El otro conjunto de procesos económicos que resulta necesario considerar, es el referido a los efectos de orden sectorial y regional que se han derivado para las actividades primarias y de servicios a partir de la adopción del modelo de desarrollo nacional, sustentado en la apertura y vinculación al mercado intemacional, tarea que se aborda en la sección que se presenta a continuación.

\section{BASE ECONÓMICA}

El estudio y las aproximaciones que se hacen de la base economica local y regional, se concentra en dos propuestas principales: la primera de ellas, con una visión comprensiva, se interroga sobre la génesis, dirección y funcionalidad de las economías locales, por considerar que es en este nivel en donde se ubica el motor del desarrollo nacional y se logra la diversificación de la producción, el ensanchamiento del mercado y la capacidad exportadora (Jacobs, 1985). La segunda propuesta tiene un campo de análisis más preciso en sus objetivos, ya que sugiere identificar el potencial de crecimiento local y regional, la capacidad y habilidad en el uso y manejo de los recursos y, por último, el manejo optimo y eficiente en las escalas de producción. Esos propositos están ubicados en un planteamiento térico y metodológico de corte neoclásico y keynesiano (Tiebout, 1962), y sujeto a la condición ceteris paribus propio del análisis economico ortodoxo. En ambos casos el objeto es de explicar cómo la producción total (Q) y la demanda $(\mathrm{Y})$, tienen su potencial de crecimiento en los niveles de la demanda externa de bienes y servicios producidos en una ciudad o region.

Si bien es cierto que ambas propuestas difieren en la profundidad con la que se aborda el estudio de la estructura y el funcionamiento de la base económica local, ambas sostienen que la capacidad y potencial para ampliar la base económica local está en la exportación. La mayor parte de las economías fronterizas tienen como característica el contar con dos componentes en su funcionamiento, como son la propensión a exportar, y una elevada propensión a importar bienes de consumo como resultado de su elevado nivel de exposición con el exterior del país, y cuyo balance es regulado por las variaciones experimentadas por el tipo de cambio.

El municipio y la ciudad de Mexicali no son ajenos a los efectos derivados de su exposición con el exterior, que se manifiestan por un lado, en la balanza de intercambios comerciales, pero también en una balanza 
de movimientos migratorios y en menor medida, de capitales que impactan el funcionamiento de su economía. Los ajustes estructurales y monetarios que derivan de los desplazamientos de factores y productos son difíciles de aproximar, por lo que abordaremos el análisis de la base económica de Mexicali desde una perspectiva alterna que integre la estructura sectorial, socioprofesional y económica de la ocupación, que nos llevará a estudiar los sectores y subsectores productivos, con el propósito de identificar las modalidades y las condiciones particulares que han desencadenado y modelado la economía local, así como establecer el potencial de desarrollo que la estructura productiva tiene en términos de su diversificación, especialización e integración que repercute en su capacidad de exportacion.

\section{La especificidad de lo fronterizo}

La especificidad de la base económica del municipio de Mexicali está estrechamente ligada a su ubicación fronteriza y al desarrollo tecnológico que impactó a la agricultura mundial desde finales del siglo XIX, con el control y manejo de los ríos para el uso agrícola. En el caso del río Colorado, permitió la construcción y desarrollo de los sistemas de irrigación de Yuma en Arizona, del valle de Imperial en Califomia y el valle de Mexicali en Baja California, que fue determinante en el origen y posterior desarrollo de la ciudad de Mexicali. Económicamente, esta posibilidad encontró un campo fértil en la afluencia de capitales del este y sudoeste de los Estados Unidos que se daba en ese momento, y que en la colonización del desierto del Colorado proporcionó una oportunidad económica y un medio para darle continuidad a dicho proceso de colonización, emprendido en ese país al amparo de la Homestead Act de 1862 (Padilla y Piñera, 1991:149).

Del lado mexicano, la promulgación de la Ley sobre colonización de terrenos baldíos en 1857, sentó las condiciones para la colonización que se dio posteriormente en el valle de Mexicali, y para su integración temprana a la expansión de la agricultura estadounidense del sur de California, al adquirir, la Colorado River Land Co., en 1904, 185000 hectáreas de bien raíz de Guillermo Andrade ${ }^{1}$ y, más tarde, en el mismo año, las acciones de la Sociedad de Irrigación y Terrenos de Baja California que le daban acceso a los derechos de agua. Una acción semejante se había

1 "En 1986 Andrade obtuvo del presidente Lerdo de Tejada títulos de propiedad por 55 secciones de terreno, en la parte meridional del río Colorado, que denominó Colonia Lerdo" (Padilla y Piniera, 1991:174). 
emprendido previamente por el mismo grupo de inversionistas en el estado de California, de tal manera que la California Development Co. se posicionó en el valle de Imperial. Con esto, ambas filiales controlaban en esas condiciones el agua y la tierra de los dos valles, creando condiciones para el surgimiento de una región agrícola internacional.

Este proyecto, que ligaba técnicamente las dos poblaciones, fue diferenciándolos económica y socialmente debido a las condiciones particulares de cada uno de los países, a lo que contribuyó la presencia de la frontera. Como resultado de esto último, identificamos tres aspectos relevantes: primero, la especialización diferente de la producción de cada uno de los valles; segundo, el flujo de trabajadores agrícolas entre ambos países como expresión de las diferencias laborales, políticas y salariales que posibilitaba la frontera en su contigüidad, y; tercero, un patrón de asentamiento de la población diferente en ambas regiones (Estrella, 1980:18).

La frontera tiene un papel determinante en la configuración de la base económica local en lo que corresponde a la especialización agrícola en cada uno de los valles, y explica las migraciones cotidianas que hoy día hacen los trabajadores agricolas mexicanos residentes en Mexicali para trabajar en el valle de Imperial. En el momento en que en la frontera se comienzan a establecer controles al flujo de población entre los dos países, el patrón de asentamiento en la gran región agrícola experimenta cambios; como ejemplo de esto, en 1927, se estimaba que alrededor de 7000 mexicanos se asentaban en el valle de Imperial ${ }^{2}$ laborando en las actividades agrícolas. En 1975 se estimaba que el número de trabajadores agrícolas residentes en Mexicali y su valle que laboraban en la agricultura de California, era de aproximadamente $5500 .{ }^{3}$ Un estudio más reciente estimaba una cifra semejante, destacando la importancia que tienen los trabajadores ocupados en el sector agrícola en el cruce Mexicali-Caléxico. Las diferencias en el costo deI suelo, del agua y del trabajo en ambos valles, así como las formas de organización en la operación de cada uno de ellos, han llevado a dos patrones de cultivo diferentes y al flujo de trabajadores como elemento de regulación en la producción agrícola regional. De esta

2 "A mediados de la década de los veinte, había una proporción importante de mexicanos que se encontraba trabajando en el valle de Imperial. En 1927 se estimaba que había alrededor de 7000 mexicanos en el área, inhibiendoel crecimiento de la población en el lado mexicano" (Samaniego, 1991).

3 En 1975 se estimaba que había 21178 trabajadores mexicanos residentes en Baja California que laboraban cotidianamente en California. De este total, 6019 eran residentes en Mexicali. Datos del INS, para 1978, 1980 y 1982, estimaban que aproximadamente $90 \%$ del total de transmigrantes Mexicali-Caléxico estaban ocupados en la agricultura. (Espinoza, 1983:20). 
manera, la agricultura, aunque ba reestructurado y reducido su participación proporcional en la economía de Mexicali, mantiene su importancia en términos de ocupación en las actividades económicas, en virtud de que no refleja únicamente la dinámica y composición de la actividad del lado mexicano, sino también en parte del sur de California a la que articulan con sus movimientos los trabajadores.

El desarrollo de Ios otros sectores ha sido más reciente y, a diferencia de lo que sucedió con la agricultura que fue activada por la localización fronteriza, la industria regional durante muchos años fue inhibida por la presencia de la frontera. En el caso de Mexicali, el crecimiento industrial se desalentó por la amputación de sus áreas de mercado, y por las regulaciones arancelarias y no arancelarias que caracterizan a estas economías regionales en el marco de economías cerradas, ${ }^{4}$ y también por las regulaciones específicas a las que el régimen de zona libre sometió a las actividades productivas locales que, junto con el costo de transporte, limitaron la internación de productos regionales al resto del país, evitando con esto, el aprovechamiento de las economías de escala y las posibilidades de competir en mercados más agresivos. ${ }^{5}$

Sández (1988), señala cómo, hasta mediados de los años veinte, es cuando el valle de Mexicali empieza a registrar actividades industriales, destacándose la industria cervecera, que junto con la producción de vinos y licores en la zona oeste del estado hicieron que se estableciera la primera restricción al perímetro libre en 1934 (Sández, 1988:185-198). El mismo autor señala cómo, desde finales de la década de los treinta, con la expropiación de las tierras en el valle de Mexicali, aparecen condiciones favorables para el crecimiento de una economía local, en donde la capitalización del campo y la sustitución local de algunos productos que anteriormente se importaban, contribuyeron a su crecimiento. Sin embargo, esta activación de la producción local se limitó por dos aspectos: el primero de ellos, porque la concurrencia de productos de importación que se internaban a la región, al amparo del régimen de la zona libre, competían ventajosamente y limitaban las "... posibilidades de realización del potencial correspondiente a la capacidad instalada en la economía" de Mexicali

\footnotetext{
4 Se refiere a las limitaciones que para la economía espacial tienen las localizaciones fronterizas en términos del tamaño del mercado; según este razonamiento, las barreras arancelarias y no arancelarias distorsionan y limitan el desarrollo y expansión del mercado. (Giersch, 1949:87-97; Hansen, 1977:2-9).

5 Se refiere a las limitaciones impuestas a la producción que se realizaba dentro del territorio de la zona libre, debido al uso de insumos internados a la región al amparo del régimen especial. (Teutli, 1986:129-137).
} 
(Sández, 1988:189). El segundo, ya señalado, se refiere a las reglamentaciones aduanales que limitaron la internación de productos regionales al centro del pais, ${ }^{6}$ con lo que se restringía el tamaño de mercado.

En medio de este ambiente restrictivo para la expansión del mercado al que se enfrentó la industria, los sectores que lograron crecer y consolidarse fueron el comercio y los servicios, comportamiento que no es ajeno a lo que sucedió en la mayoría de las otras localidades fronterizas del norte. Sin embargo, con todas estas limitaciones, la industria en Mexicali experimentó una temprana presencia en los subsectores ligados a la transformación de recursos naturales y materias primas, que fueron en su momento, de los más importantes de Baja Califomia, de tal manera que el municipio contaba en 1955 con $80.0 \%$ de la producción industrial del estado y reunía $75.0 \%$ de la inversión industrial de Baja California. ${ }^{7}$ (Zazueta, 1982, cit. en Sández, 1988).

La década de los sesenta marca la transformación de la estructura productiva local como resultado de una actividad industrial, la cual continúa desarrollándose alrededor de la industria de alimentos y la presencia incipiente de una industria de autotransporte con inversiones en la misma zona. Para la segunda mitad de la década de los sesenta, comienzan a instalarse las primeras industrias maquiladoras en la ciudad, que le dan un sesgo muy importante a la estructura productiva local, diversificándola e introduciendo cambios en las formas y prácticas de organización que preludia el proceso de internacionalización de la producción. Esto es, el pasaje de una producción activada desde la perspectiva de la oferta, y el determinismo que convierte a la producción agrícola local en el sustento del desarrollo de las otras actividades y sectores, hacia una industria sustentada en la demanda internacional y cada vez menos dependiente de los recursos naturales.

Referente al primer señalamiento, destaca cómo el sector industrial regional se desarrolla alrededor de la transformación de alimentos y más tarde de la industria del autotransporte, dado que, como ejemplo de este cambio, se observan variaciones significativas en el valor agregado entre los subsectores industriales en el periodo 1960-1975, En 1960 la industria de alimentos participaba con $44.4 \%$ y en 1975 la proporción descendió a

6 Aún hoy día esta situación se mantiene; como ejemplo de esto, si consideramos las exportaciones del estado de Baja California como integradas por el valor agregado que se exporta - vía la industria mauiladora-, el valor de los bienes y servicios que se exportan al extranjero y el valor de los bienes que se internan al centro del país, las proporciones son, respectivamente, de $61.1 \%, 35.6 \%$ y 3.3\%, para el año de 1994. (Estadisticas económicas de Baja Califomia, 1995).

7 En contraste con el tipo de empresas agrícolas que se instalaron en el valle de Imperial que eran pequeñas (Zazueta, 1982, cit, en Sández, 1988:190). 
19.9\%. En contraste, de tener una participación mínima en el primero de estos años, la producción de material eléctrico y de transporte, participaron con el $13.5 \%$ y $32.3 \%$ respectivamente, del total en 1975 .

En relación con la instalación de maquiladoras en Mexicali, se debe advertir que, como sucedió en la mayoría de las localidades de la frontera, introdujo cuando menos dos elementos en la base económica de las principales localidades fronterizas. En el marco de una reestructuración continua a lo largo de casi tres décadas del programa maquilador, dicho programa insertó sectores ajenos al desarrollo histórico de las economías locales, acelerando con ello la modernización en la industria de la región y su articulación con el mercado internacional. Para 1973 ya había 39 empresas que ocupaban cerca de 7000 empleados y trabajadores; para 1994 el número de establecimientos había pasado a 120 y el de empleados en cerca de 20000 . El segundo aspecto, es que estas industrias, al instalarse en las principales ciudades fronterizas, modificaron el paisaje industrial y comenzaron a introducir cambios en las prácticas organizacionales, que fueron transformando y modernizando la industria local y la cultura de los empresarios locales.

De todo lo anterior, lo que nos parece significativo señalar de la base económica de Mexicali, es la conformacion de tres segmentos productivos: un sector agrícola, que mantiene su presencia en la economía local; una serie de actividades de los sectores industrial, comercio y de servicios sustentado en el mercado regional, en su mayoría pequeña y mediana industria; y tercero, un sector de industrias orientadas a la exportación y prácticamente sin eslabonamientos industriales a nivel regional. Estos segmentos de la estructura productiva local son el potencial de desarrollo de la base económica, cuyo principal reto es lograr una articulación entre ellos que haga más competitiva a esta economía y la conduzca a la multiplicación de las exportaciones.

\section{El perfil de exportación}

El análisis de la base económica local, lo hacemos utilizando principalmente la variable ocupación para estimar indirectamente la estructura productiva local. El método de análisis que usamos es la estimación de los cocientes de localización, que es una metodología que tiene la virtud de estandarizar las proporciones en la ocupación, según las diversas clasificaciones que se analizan de la variable. Los resultados se interpretan en términos de que aquellos valores superiores a la unidad son sectores, categorías o subsectores con una participación excedentaria que se asume como la capacidad o potencial de exportación. 
Con la metodología descrita y con la interpretación general establecida, se busca deducir los sectores claves y el análisis de la estructura de producción del municipio de Mexicali, lo cual hacemos con una perspectiva longitudinal de los cocientes de localización de diferentes modalidades de la variable ocupación. El propósito principal es apreciar la capacidad y potencial de exportación que tiene la economía local en el marco de las tendencias de cambio de los últimos cuarenta años, para lo cual realizamos tres aproximaciones que manejan, por un lado, la estimación de la variable de ocupación por lugar de residencia ${ }^{8}$ y, por el otro, la ocupación por lugar de trabajo. Esta distribución estimada de manera puntual y en el marco de comparación territorial, consideramos que puede conducir a una aproximación al comportamiento de la base económica local de una manera indirecta, la que en su conjunto expresará las estrategias locales e internacionales de las firmas que coinciden en Mexicali en un momento dado (Fischer, 1994:6). En este sentido, creemos que los cambios que identificamos a diferentes niyeles de desagregación, expresan el grado de apertura y la capacidad de articulación que tienen las actividades económicas locales con el exterior.

\section{a) Cambios sectoriales}

Analizando los cambios experimentados entre 1950 y 1990 en la población económicamente activa para los tres sectores productivos del municipio de Mexicali, se identificó la distribución de la ocupación sectorial de la poblacion, la cual es presentada en la figura 2.

Esta tendencia en el cambio de la distribución sectorial del empleo del sector primario al terciario, ha sido un proceso generalizado en la mayor parte de las sociedades occidentales durante el presente siglo, confirmándose en México y en particular en Mexicali. Sin embargo, en el caso específico de Mexicali, este cambio se produce manteniéndose un sector primario que exporta en los últimos años hortalizas y otros productos, que lo mantiene y convierte en una de las principales fuentes de exportación para el estado de Baja California. En 1994, las exportaciones de bienes primarios de dicho estado representaban aproximadamente una tercera parte del valor agregado generado en la industria maquiladora.

8 Se refiere al uso que hacemos de las variables de ocupación para la PEA, registrada según lugar de residencia en los Censo de población y vivienda.

9 Se refiere a los registros de la población ocupada en el lugar de trabajo, información que se consigna en los Censos económicos. 


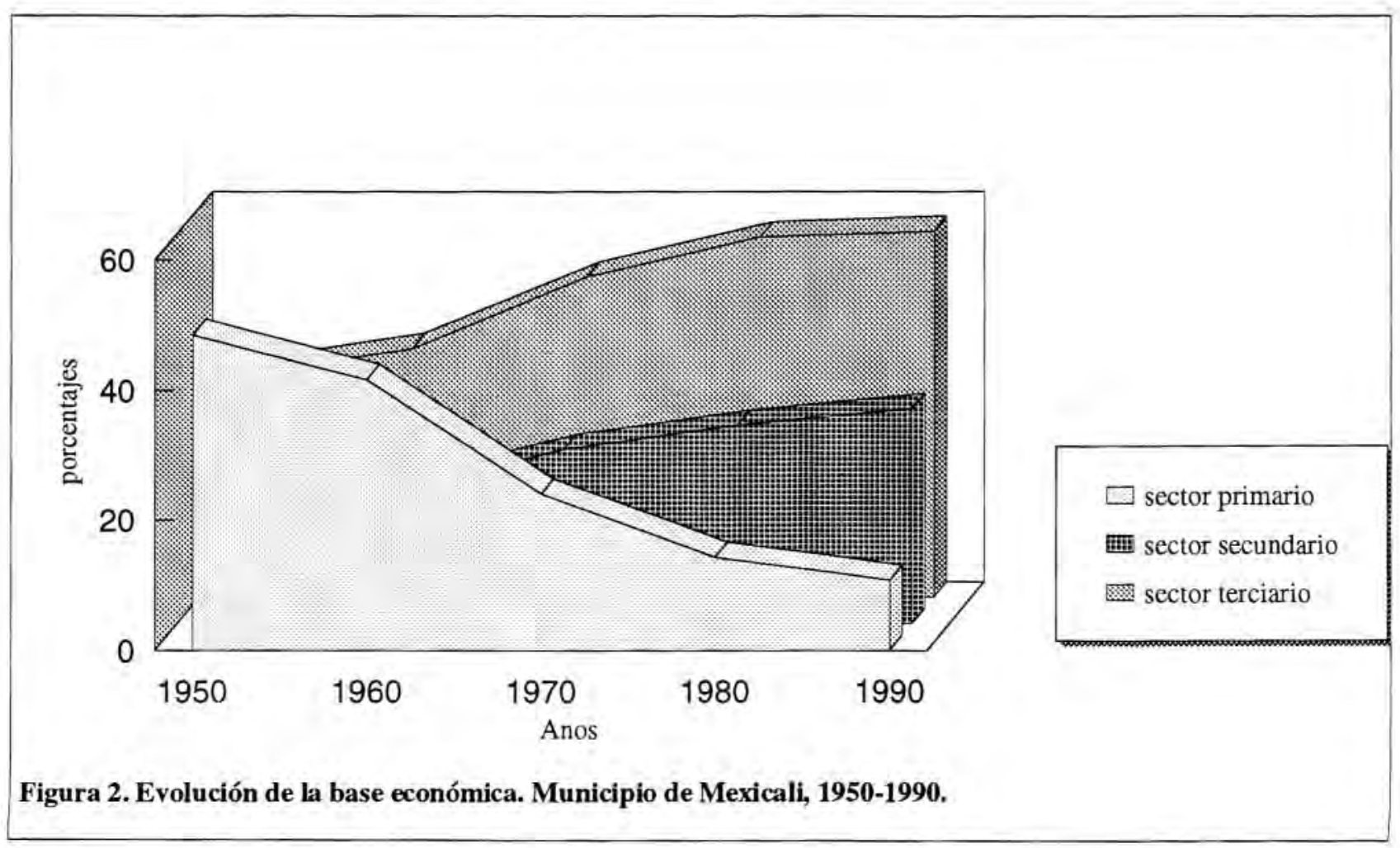


En términos de ocupación sectorial, la comparación de los cocientes de localización obtenidos nos llev6 a los resultados que consigna el cuadro 2.1. Los valores muestran como sector representativo de Mexicali al sector primario, y en particular a la agricultura, que es la actividad predominante en este sector en el municipio.

\section{CUADRO 3. Cocientes de localización PEA por sector de actividad municipio de Mexicali 1950-1990.}

\begin{tabular}{lccc}
\hline Año & Primario & $\begin{array}{c}\text { Sectores } \\
\text { Secundario }\end{array}$ & Terciario \\
\hline 1950 & 1.27 & 0.69 & 0.77 \\
1960 & 1.31 & 0.75 & 0.79 \\
1970 & 2.27 & 0.56 & 0.62 \\
1980 & 1.41 & 0.87 & 0.96 \\
1990 & 1.73 & 0.82 & 0.96
\end{tabular}

Fuente: Cálculos realizados a partir de los Censos generales de población y vivienda, 1950-1990, INEGI.

El cálculo de los cocientes de localización se formaliza de la manera siguiente:

$C e=\frac{p o M X \text { si/noMX }}{p o B C \text { si ptoBC }}$

los resultados mayores de 1 , reflejan una especialización local o regional, una capacidad o potencial de exportación.

En donde;

$\mathrm{Ce}=$ cociente de especialización.

poMx si = población económicamente activa en el sector $\mathrm{i}$, en el municipio de Mexicali.

poMX = población económicamente activa en el total de los sectores en el municipio de Mexicali.

poBCsi = población económicamente activa en el sector $\mathrm{i}$, en el estado de Baja California.

poBC $=$ población económicamente activa en el total de las sectores en el estado de Baja California. 
En relación con los otros dos sectores, se aprecia una emergencia gradual en los sectores secundario y terciario que, debido al método utilizado, es subvalorado por el crecimiento acelerado que en estas actividades ha experimentado el municipio de Tijuana. Esto significa que la actividad representativa, en términos de su capacidad exportadora en el municipio de Mexicali, continúa estando en la agricultura; mientras que el crecimiento gradual en la industria, el comercio y los servicios, aunque ha incrementado, se mantiene por debajo de los cambios experimentados en el estado de Baja California en su conjunto. ${ }^{10}$

\section{b) Cambios socioprofesionales}

La transformación sectorial no aporta suficientes indicios de los cambios cualitativos experimentados por la base económica local. En este sentido, las modificaciones representadas en la distribución socioprofesional de la PEA, nos parece un medio pertinente para aproximar cambios en el proceso de modernización de la estructura económica que está muy expuesta al exterior.

De los resultados presentados en el cuadro 4, podemos destacar dos observaciones: la primera, es la presencia de un importante sector agrícola que, al compararse con la proporción estatal, muestra una participación creciente en relación con el conjunto de Baja California, y confirma el crecimiento acelerado que los industriales y de servicios han tenido en el resto del estado. La segunda observación se refiere a un crecimiento sistemático en la participación de profesionales y técnicos, funcionarios y directivos, y de trabajadores en servicios.

En suma, la base económica local tiene su mayor capacidad exportadora en términos de ocupación en las actividades agrícolas, en cuyo total se incluyen tanto a los residentes y trabajadores en Mexicali, como a los residentes de Mexicali que laboran en la agricultura del sur de California, para quienes esta ciudad se convierte en un área-dormitorio y se beneficia de la derrama de ingresos procedentes del exterior. También es destacable que la base económica local experimenta un proceso de cambio en términos de su calificación, dado el incremento en la ocupación a niveles técnico y profesional, así como un crecimiento sostenido en los cuadros directivos; esto representa un potencial en puestos de conocimiento "acumulado" y una tendencia creciente a la ocupación en las actividades de servicios.

\footnotetext{
${ }^{10}$ Por el método de análisis utilizado, los resultados en el sector secundario y terciario en términos de los cocientes de localización, manifiestan el crecimiento reciente y rápido de estos sectores, en particular en Tijuana.
} 


\section{CUADRO 4. Cocientes de localizacion. PEA por posición en el empleo. Municipio de Mexicali.}

\begin{tabular}{lllllllll}
\hline Ańo & $\begin{array}{c}\text { Profes. } \\
\text { técnico }\end{array}$ & $\begin{array}{c}\text { Funcio. } \\
\text { direct. }\end{array}$ & $\begin{array}{c}\text { Admini. } \\
\text { oficina }\end{array}$ & Comer. & $\begin{array}{c}\text { Trabaj. } \\
\text { agric. }\end{array}$ & $\begin{array}{c}\text { Supervi. } \\
\text { industria }\end{array}$ & $\begin{array}{c}\text { Trabaj. } \\
\text { servic. }\end{array}$ \\
\hline & & & & & & & & \\
1950 & 0.74 & 0.79 & 0.81 & 0.73 & 1.23 & 0.67 & 0.71 \\
1960 & 0.84 & 0.62 & 0.70 & 0.81 & 1.32 & 0.74 & 0.77 \\
1970 & 0.90 & 0.79 & 0.80 & 0.83 & 1.41 & 0.83 & 0.74 \\
1980 & 0.97 & 0.97 & 1.13 & 0.86 & 1.38 & 0.84 & 0.90 \\
1990 & 1.10 & 0.99 & 0.87 & 0.92 & 1.76 & 0.82 & 0.89 \\
\hline
\end{tabular}

Fuente: Cálculos realizados a partir de los Censos generales de población y vivienda, 1950-1990, INEGI.

El cálculo de los cocientes de localización se formaliza de la manera siguiente:

$C e=\frac{p o M X \text { si } / \text { noMX }}{p o B C \text { si proBC }}$

los resultados mayores de 1, reflejan una especialización local oregional, una capacidad o potencial de exportación.

En donde;

$\mathrm{Ce}=$ cociente de especialización

poMx si = población económicamente activa en la categoría socioprofesional i, en el municipio de Mexicali.

poMX = población económicamente activa en el total de las categorias socioprofesionales en el municipio de Mexicali.

poBCsi = población económicamente activa en la categoría socioprofesional i, en el estado de Baja California.

poBC = población económicamente activa en el total de las categorías socioprofesionales en el estado de Baja Califormia.

\section{c) Cambios en la estructura productiva}

Los cambios en la estructura productiva se estimaron en relación con la ocupación en los subsectores que registran los censos económicos de 1986, 1989 y 1994. Metodológicamente, esta información y su clasificación en subsectores, constituye un corte más fino que facilita acercamos a la estructura productiva local.

Los resultados de los cocientes de localización por sectores de actividad, facilitaron la identificación de la estructura en la ocupación y su nivel de diversificación con respecto al resto de Baja Califomia. Los valores 


\section{CUADRO 5. Coeficientes de localización. Establecimientos y personal ocupado. Municipio de Mexicali.}

\begin{tabular}{llll}
\hline $\begin{array}{l}\text { Clave } \\
\text { Sub-sector }\end{array}$ & 1985 & 1988 & 1994 \\
\hline 31 & 0.89 & 0.70 & \\
32 & 1.46 & 1.59 & 1.20 \\
33 & 0.32 & 0.24 & 0.84 \\
34 & 0.69 & 1.29 & 0.28 \\
35 & 0.80 & 0.88 & 1.49 \\
36 & 0.79 & 1.25 & 0.48 \\
37 & 1.39 & 0.34 & 1.13 \\
38 & 1.00 & 1.12 & 1.96 \\
39 & 0.93 & 1.03 & 0.93 \\
61 & 1.24 & 1.45 & 0.05 \\
62 & 0.98 & 1.08 & 1.15 \\
81 & 0.88 & 0.75 & 1.15 \\
82 & 0.96 & 0.47 & n.d. \\
83 & 0.76 & 1.55 & 1.04 \\
92 & 0.95 & 1.08 & 1.10 \\
93 & 0.82 & 0.92 & 1.07 \\
94 & 0.58 & 0.80 & 0.99 \\
95 & 1.01 & 0.91 & 0.87 \\
96 & 1.00 & 1.20 & 1.00 \\
& & 1.27 \\
\hline
\end{tabular}

Fuente: Cálculos realizados a partir de los Censos económicos de 1986, 1989 y 1984, INEGI.

El cálculo de los cocientes de localización se formaliza de la manera siguiente:

$C e=\frac{p o M X s / / \text { nom } x}{p o B C s s / p s o B C}$

los resultados mayores de 1, reflejan una especialización local o regional, una capacidad o potencial de exportación.

En donde: $\mathrm{Ce}=$ cociente de especialización.

poMx si = personal ocupado en los establecimientos del subsector I, en el municipio de Mexicali.

poMX = personal ocupado en los establecimientos del total de subsectores del municipio de Mexicali.

poBC si = personal ocupado en los establecimientos del subsector $I$ en el estado de Baja California.

poBC $=$ personal ocupado en los establecimientos del total de subsectores del estado de Baja California.

${ }^{a}$ Se estandarizaron los subsectores para el periodo de estudio por medio de la exclusión de dos sectores, como son el de la industria de la construcción por estar ligada a la coyuntura y el de la generación y distribución de energía eléctrica, que está muy concentrada por la empresa paraestatal que presta el servicio. 
obtenidos llevan a identificar dos grupos (ver cuadro 5). El primero está constituido por las industrias extractivas (29) y manufactureras $(32,34$, $36,38,39)$; la actividad extractiva se concentró en una explotación corporativa de reciente instalación fuera de la ciudad y con un peso específico alto en este subsector en el estado.

El segundo grupo está integrado por dos componentes: el de comercio y los servicios. El comercio ( 61 y 62) mantiene un peso significativo que en mucho responde al papel central que juega la ciudad y que fomenta la actividad comercial en sus expresiones de mayoreo y menudeo. El sector de servicios, por otra parte, se caracteriza por el desarrollo reciente de actividades de apoyo a las demás actividades productivas y que responde a las nuevas tendencias de organización industrial. Tal es el caso de una actividad de alquiler de inmuebles, en donde se incluyen la renta de naves industriales (83), mantenimiento y reparacion (96) y en la presencia de una ocupación importante a nivel local en actividades educativas, de investigación y servicios especializados (92) (ver figura 3).

Es evidente la necesidad de realizar un análisis más detallado de la base económica local en donde se incluyan, junto con la información de ocupación, la información de mercados, de variables monetarias y de impacto fiscal, derivado de la localización fronteriza y los recientes cambios en la política económica nacional. Sin embargo, el análisis que presentamos con base en la evaluación somera que hicimos de variables de ocupación, arroja pistas sobre algunas tendencias que están presentes en las actividades de la economía mexicalense. Estas pistas, junto con los resultados de algunos estudios amplios en proceso, ${ }^{11}$ en los que emerge la especialización de los municipios, nos llevan a plantear como conclusión de este análisis que hay cambios en proceso en la economía de Mexicali, en donde ha perdido peso la transformación de alimentos y recursos naturales que constituían el eje en el pasado, y en donde las manufacturas están experimentando uno de diversificación en el que se integran nuevos subsectores. En el comercio y los servicios, se distingue el peso que adquieren actividades de apoyo y conocimiento acumulado y especializado en el total de las actividades, como expresión de una transformación cualitativa en la planta productiva. Planta productiva en donde la generación de energía eléctrica y su exportación, junto con la disponibilidad de agua, le dan una ventaja locacional al municipio en relación con el resto del estado de Baja California.

" Es el caso del estudio: "Baja California hacia la competitividad", editado por el Gobierno del estado de Baja California (1995). 


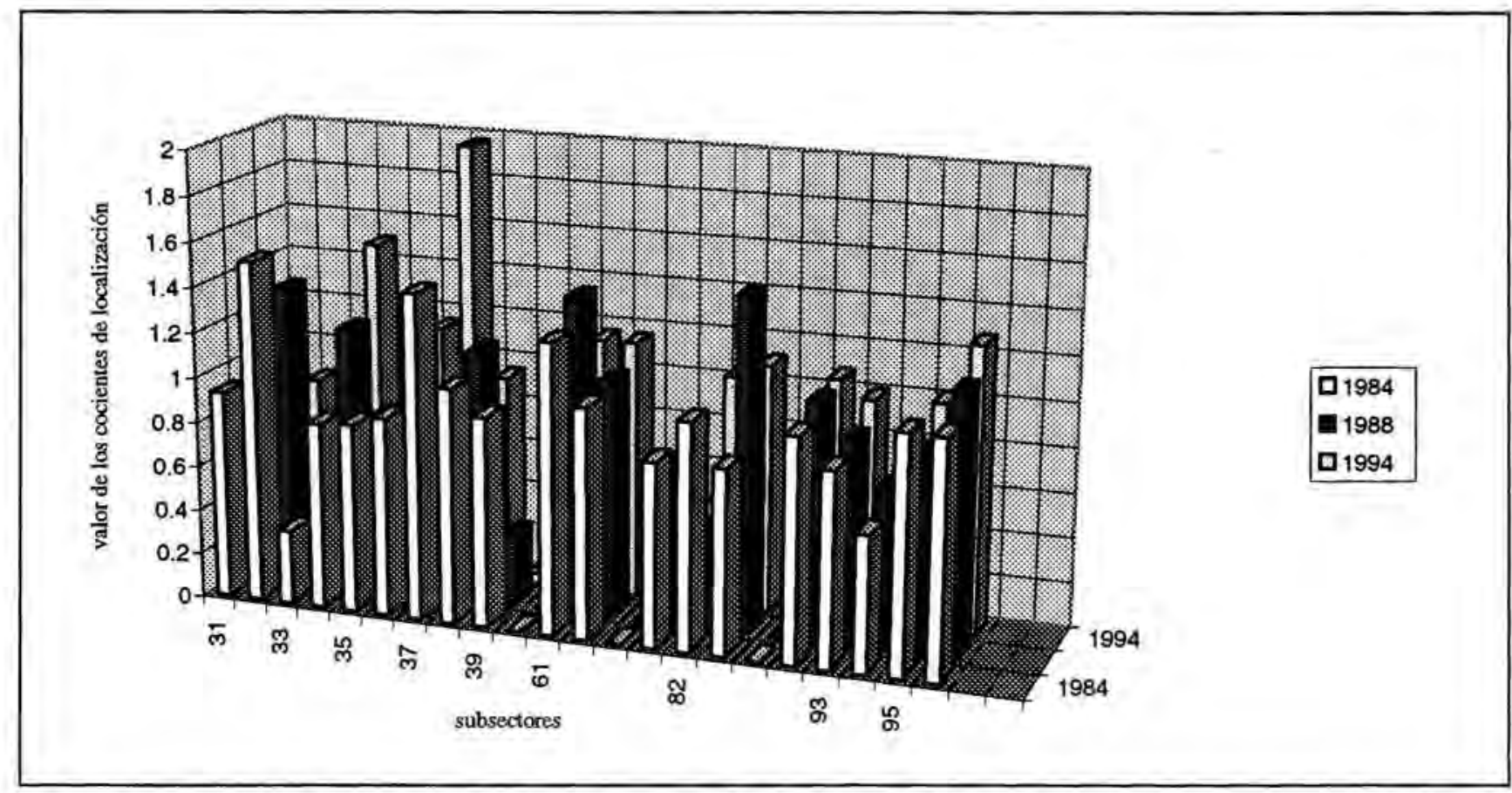

Figura 3. Cocientes de localización de las actividades productivas del municipio de Mexicali, B.C. 1985-1994. 


\section{CONCLUSIONES}

Las dos líneas de análisis que se han presentado, han permitido identificar los elementos generales con los que se puede definir la coyuntura en la que se encuentra actualmente la ciudad de Mexicali.

En el ámbito demográfico, la ciudad ba dado muestras de estar iniciando un nuevo ciclo de reactivación de la velocidad del crecimiento social de su población. La intensidad y duración que llegue a adquirir esta renovada capacidad de atracción de migrantes que se ha observado durante la segunda mitad de la década de los ochenta, en principio puede asumirse que estará vinculada -como sucedió en el pasado- con el aprovechamiento que se haga de las ventajas locacionales y de capital humano de las que dispone esta localidad.

En función de lo anterior, conviene delimitar la problemática de la economía de la zona en dos puntos. El primero de ellos es la conformación de una economía local en tres segmentos que son: la base agrícola con evidencias de reestructuración y reorganización; las actividades en los sectores de industria y servicios de origen local, que han entrado en un proceso de restructuración por las condiciones recientes en que la apertura del país ha afectado los flujos de bienes y servicios, y que han llevado a cambios en las regulaciones locales derivadas del régimen de zona libre; y por último, un sector industrial constituido princípalmente por la industria maquiladora, que aprovechando la contigüidad fronteriza ha crecido durante los últimos años.

En conjunto, se advierte que existe una estructura diversificada que en la práctica está desarticulada, y cuyo reto principal es buscar una vinculación horizontal que integre realmente a estos tres segmentos y facilite una multiplicación de las exportaciones.

El segundo aspecto se refiere a los cambios detectados en la ocupación y en su distribución, que representa la capacidad y el potencial humano que tiene la ciudad para activar la economía. Aquí se tienen que destacar dos aspectos: por el lado de la oferta, la presencia de cuadros altos y medios que ha venido creciendo en los últimos años y que reflejan una mayor calificación regional, pero también un incremento en la oferta de estos empleos que pone de manifiesto un proceso de cambio en la planta productiva local; los resultados de 1986 a 1994 parecen confirmar esto. Y por otro lado, la diversificación en la industria y en los servicios -en donde destaca la ocupación en las empresas de servicios especializados, servicios educativos y de investigación-, parece apuntar a una actividad productiva en el conocimiento acumulado, lo cual concuerda con las nuevas formas de funcionamiento de las organizaciones económicas. 


\section{BIBLIOGRAFÍA}

Baja California hacia la competitividad. 1995, Gobierno de estado de Baja California. CETYS.

CONAPO. 1992. Indicadores socioecómicos e indice de marginación municipal 1990, Consejo Nacional de Población, México, D.F.

CORONA, R, 1991. "Principales características demográficas de la zona fronteriza del norte de México", Frontera norte, vol.3, núm. 5, enero-junio, El Colegio de la Frontera Norte, Tijuana, B.C.

ESPINOZA, J.F. 1983. "Condiciones de trabajo y situación socioeconómica del trabajo agrícola transmigrante del valle de Imperial". Cuadernos de Ciencias Sociales, Serie 1, núm. 5, nS-UABC.

Estadísticas económicas de Baja California. 1995. Secretaría de Desarollo Económico, Gobierno del estado de Baja California.

ESTRELLA, G. 1980. "El origen de la región de Ios valles de Mexicali e Imperial, desde la perspectiva de las relaciones sociales", Cuadernos de Ciencias Sociales, serie 4, núm. 1. IIS-UABC.

FISCHER, A. 1994. Industrie et espace geographique. MassonGeographie, París.

GIERSCH, H. 1949. "Economic Union Between Nations and the Locations of Industries", Review of Economic Studies, vol. 17, núm. 43.

GUTIÉRREZ, M. 1944. "Las regiones de México ante el thc", Comercio exterior, vol. 44, núm. 11, Banco Nacional de Comercio Exterior, S.N.C., México, D.F.

HANSEN, N, 1977. "The Economic Development of the Border Regions", Growth and Change, vol. 8 (4).

JACOBS, J. 1985. Cities and the Wealth of Nations, Priciples of Economic Life. Vintage Books, Nueva York.

MONTERRUBIO, L., R. Flores y M. Armada. 1993. "La transición demográfica de México por grupos de entidades federativas a partir de sus tasas brutas de natalidad y mortalidad, 1940-1990". IV Conferencia Latinoamericana de Población. La Transición Demográfica en América Latina y el Caribe, vol. II, ABEP-CELADEIVSSP-SOMEDE-INEGI-IISUNAM, México, D.F.

PADILLA, C.A. y R.D. Piñera. 1991. "El surgimiento de Mexicali", Mexicali una historia, UABC.

SAMANIEGO, M.A. 1991. "El desarrollo económico durante el gobierno de Abelardo L. Rodríguez, 1924-1928”. Mexicali una historia, UABC. 
SÁNDEZ, A. 1988. "El proceso de industrialización en Baja California", Estudios Fronterizos, vol. 6, núm. 15-16, IIS-UABC.

TEUTLI, O.G. 1986, "La política federal de desarrollo fronterizo: análisis y perspectivas". Estudios Fronterizos, vol. IV, núm. 10-11. IISUABC.

TIEBOUT, Ch. M. 1962. The Community Economic Base Study, CED, Nueva York.

ZAZUETA, C. 1982. Mexicali: su historia, la ciudad, sus actividades y sus hombres, CBNIET-STPS, México.

ZENTENO, R. y R. Cruz. 1988. "Un contexto geográfico para la investigación demográfica de la frontera norte", Estudios demográficos y urbanos, vol. III, núm. 3 (9), septiembre-diciembre, El Colegio de México, México, D.F. 